\title{
Exploiting tumor metabolism: challenges for clinical translation
}

\author{
Matthew G. Vander Heiden
}

Koch Institute for Integrative Cancer Research, Department of Biology, Massachusetts Institute of Technology, Cambridge, Massachusetts, USA. Dana-Farber Cancer Institute, Boston, Massachusetts, USA.

\begin{abstract}
The metabolism of cancer cells differs from most normal cells, but how to exploit this difference for patient benefit is incompletely understood. Cancer cells require altered metabolism to efficiently incorporate nutrients into biomass and support abnormal proliferation. In addition, the survival of tumor cells outside of a normal tissue context requires adaptation of metabolism to different microenvironments. Some existing chemotherapies target metabolic enzymes, and there is a resurgent interest in developing new cancer drugs that interfere with metabolism. Success with this approach depends on understanding why specific metabolic pathways are important for cancer cells, determining how best to select patients, and developing technologies for monitoring patient response to therapies that target metabolic enzymes. The articles in this Review series address these issues, with a focus on how altered metabolism might influence tumor progression and how this knowledge might inform the use of new therapies targeting cancer metabolism. Emerging biomarker strategies to guide drug development are also highlighted.
\end{abstract}

\section{Introduction}

Cells have evolved complex regulatory mechanisms to adapt metabolism to match physiological states (1). Cancer cells co-opt this normal regulation to fuel inappropriate cell proliferation and support survival in abnormal tissue contexts. As a result, the metabolism of tumor tissues differs from that of the normal tissues from which cancer arises (2-4). Differences in metabolism represent some of the first known variations identified between cancer cells and normal cells (5), yet the advantage of altered metabolism for tumors remains a topic of intense study with important clinical implications.

\section{Cancer cells exhibit increased nutrient uptake}

Many cancer cells increase glucose uptake, but instead of oxidizing most of this glucose to efficiently generate ATP by oxidative phosphorylation, they instead ferment the excess glucose to lactate. This phenomenon is observed even in the presence of oxygen, and is referred to as the Warburg effect or aerobic glycolysis $(2,3,5,6)$. Previously, aerobic glycolysis was suggested to be a consequence of mitochondrial damage (7) or an adaptive response to tumor hypoxia (8). However, mitochondria remain functional in most tumors, and aerobic glycolysis is observed in cancer cells independent of oxygen levels $(3,6)$. In fact, numerous studies have described a key role for mitochondrial function in cancer, and it has been suggested that oxidative phosphorylation remains an important source of ATP for many tumor cells (3, 9, 10). Nevertheless, increased aerobic glycolysis is characteristic of many cancers, and how this metabolic phenotype benefits tumor cells is a topic of debate.

Aerobic glycolysis may allow individual cancer cells to increase production of macromolecules and facilitate the construction of new cells. In support of this idea, aerobic glycolysis is a feature of many rapidly proliferating normal tissues and microorganisms (6). ATP is necessary to support macromolecular synthesis, but

Conflict of interest: The author is a consultant and scientific advisory board member for Agios Pharmaceuticals.

Citation for this article: J Clin Invest. 2013;123(9):3648-3651. doi:10.1172/JCI72391. the fractional increase in ATP required to allow proliferation is likely small relative to the amount of ATP cells require to maintain homeostasis. Satisfying the metabolic needs of proliferation beyond ATP production may be one advantage of aerobic glycolysis (10). Nevertheless, generation of sufficient ATP is necessary for survival of all cells, and the relative contribution of different pathways to ATP production likely varies across cancer types and tumor contexts.

Many normal mammalian tissues rely heavily on the use of nutrients other than glucose, and consumption of alternative fuel sources is observed in some cancer cells. Glutamine is the most abundant amino acid in both serum and cell culture medium, and glutamine is an important source of nitrogen for cells (10, 11). The carbon skeleton of glutamine can be oxidized to generate ATP and can replenish TCA cycle intermediates to facilitate biosynthesis, a process termed anaplerosis. Finally, in some contexts reductive glutamine metabolism can provide carbon for lipid synthesis (12-15). Indeed, after glucose, glutamine is the nutrient most highly consumed by cancer cells in tissue culture $(11,16)$. However, emerging evidence suggests that other nutrients, including fatty acids and other amino acids, can also play key roles in some contexts (16-21).

Increased nutrient uptake is exploited in the clinic as a way to image tumors. For instance, F-18 fluoro-2-deoxyglucose PET (FDG-PET) can be used to visualize cancers. This technique serves as a measure of glucose uptake in patient tissues by coupling positron-emitting ${ }^{18} \mathrm{~F}$ to an analog of glucose that is taken up and trapped in cells by phosphorylation but is not subject to further metabolism (22). FDG-PET is most useful clinically as a staging tool and can also be used to monitor therapy response (23-25). PET scanning to monitor uptake of other nutrients has also been described in research settings $(26,27)$, and labeled glutamine and glutamate analogs are currently in clinical development (28-31). These approaches, however, are not necessarily specific to cancer cells, and a better understanding of how increased nutrient uptake supports different stages of tumor progression will be important to exploit metabolism for therapeutic benefit. 


\section{Cancer cells use different metabolic programs}

While cancer metabolism is often considered as a single entity differing from normal cell metabolism, there is evidence that tumor cells exhibit a diversity of metabolic phenotypes $(4,16-$ $18,25,32-35)$. Heterogeneous expression of metabolic genes is observed across tissue types, and the metabolic network of an individual tumor more closely resembles that of the normal tissue from which the tumor arose than it does other tumors that develop in different organ sites (4). This expression pattern may reflect the propensity of cancer cells to adapt the pre-existing metabolic network to support the altered needs of the neoplastic tissue. Indeed, where it has been studied, the metabolic phenotype of tumors is a function of both the genetic lesion driving tumorigenesis and the tissue from which the cancer arose (32).

Altered metabolism is a consequence of many genetic lesions important for cancer $(2,36)$. Expression of oncogenes promotes increased consumption of glucose, glutamine, and proteins and can reprogram metabolism to support cell growth and proliferation (18, 37-39). Increasing evidence indicates that tumor suppressor genes function in part through effects on metabolism $(19,40)$, and the combination of genetic mutations in specific tissues facilitates altered metabolic regulation to support abnormal tissue growth. Taken together, these studies suggest that multiple genetic events converge to promote proliferative metabolism in cancer cells. However, for most cancers, only a minority of cells are proliferating at any given time in patients, and little is known about how nonproliferating cancer cells adapt metabolism to survive within abnormal neoplastic tissue contexts. A better understanding of these programs will be critical to exploiting altered metabolism to eliminate tumor cells in patients.

\section{Tumor cell metabolism is influenced by external factors}

The tumor cell microenvironment can profoundly affect metabolism and influence how nutrients are metabolized (12, 37). In addition, tumors are composed of a heterogeneous mixture of cancer cells and normal cells, and symbiotic metabolic relationships have been described among cells in both normal and malignant tissue contexts (41-44). The complex interplay between genetics, microenvironment, and tissue heterogeneity is a poorly understood aspect of tumor metabolism. In addition, whole body metabolic regulation can affect tumor tissue metabolism, and patients with cancer often have perturbations in whole body metabolism (45).

Altered organismal metabolism can affect cancer outcomes, as evidenced by the relationship between cancer cachexia and poor patient survival $(46,47)$. In addition, there is a survival advantage for diabetic patients taking metformin who also have cancer, when compared with similar patients whose glucose levels are controlled by other means $(48,49)$. Powerful homeostatic mechanisms exist at the organismal level to maintain a relatively constant supply of nutrients available to both normal and malignant tissues. While the presence of cancer can influence organismal metabolic regulation, the complex relationship between this regulation and diet complicates translation of nutrient auxotrophies identified in cancer cell line cultures into patient therapies. Incorporating the intricacies of whole body metabolism and metabolic heterogeneity into our understanding of cancer cell metabolism remains a challenge for the field.

\section{Challenges to developing drugs targeting cancer metabolism}

Key questions exist regarding how to target metabolic pathways for cancer treatment (36). First, it is critical to understand in the correct physiological context how metabolic alterations provide a benefit to tumor cells, as this can provide the foundation for developing rational combinations with existing therapies. Next, how best to select patients who will respond to metabolic enzyme inhibitors remains an important issue. Therapies targeting signal transduction pathways have been most successful when patients are chosen based on tumor genetic mutations, but metabolic enzymes are not mutated in most cancers. Technical hurdles associated with measuring metabolism in vivo also complicate the development of surrogate markers for determining whether drugs targeting metabolic enzymes modify their target in tumors. Potential untoward side effects of disrupting normal tissue metabolism are also often raised as a concern, and the role of organismal metabolism in modulating tumor responses to metabolic pathway inhibition adds another layer of complexity. The collection of Review articles in this issue of the JCI addresses many of these challenges.

Several of the Reviews update our current understanding of how altered metabolism influences tumor biology. Michael Pollak touches on the interaction between organismal metabolism and cellular metabolism in reviewing the data surrounding the effects of metformin on cancer outcomes (50). He discusses ongoing trials to explore the use of metformin in cancer patients and raises issues related to optimizing drug dose, patient selection for specific therapies, and the selection of rational combinations to maximize patient benefit. This Review also explores the broader issue of how the current use of drugs with metabolic targets approved for non-cancer indications might inform how best to target cancer metabolism.

Another example of drug repurposing for cancer treatment is the use of dichloroacetate, which can act in part by interfering with metabolic reprogramming mediated by the transcription factor HIF-1. Gregg Semenza discusses the biology surrounding HIF-1 as an example of how genetic events can have pleiotropic effects leading to metabolic reprogramming and can provide an advantage to tumor cells (51). This Review also considers how tumor cells adapt to microenvironmental changes in oxygen levels and discusses regulation of angiogenesis as an adaptive response to cell stress, illustrating how physiological context can affect both metabolic regulation and tumor progression.

Ralph DeBerardinis and colleagues review differential nutrient use by cancer cells as another example of how physiological context might affect tumor biology. Their Review focuses on how glutamine is metabolized by cancer cells, but also discusses aspects of glutamine metabolism by normal tissues and how this may affect organismal metabolic changes in cancer such as cachexia (52). Potential drug targets for interfering with tumor glutamine metabolism are also covered, as are strategies to track glutamine metabolism in patients that could influence patient selection for agents targeting these pathways.

Doherty and Cleveland explore how the propensity of tumors to utilize aerobic glycolysis and generate lactate might be exploited for cancer therapy (53). This Review includes a discussion of how lactate production and consumption by different cell populations within a tumor could be involved in cancer, and how elevated lactate production alters the tumor microenvironment to favor 
malignant tissue growth. The article also discusses specific molecules and targets being considered for therapeutic intervention to capitalize on this aspect of altered tumor metabolism.

Patrick Pollard and colleagues discuss the concept of oncometabolites and how changes in metabolism can lead to altered metabolite levels that might promote transformed phenotypes (54). They examine how oncogenic changes in metabolites can directly affect enzymes and specifically discuss how altered metabolites can perturb the oxidative stress response, in line with recent literature suggesting that modified fumarate levels may allow adaptive responses to different cell environments $(55,56)$.

Another cancer-associated metabolite is 2-hydroxyglutarate (2-HG). 2-HG is produced by the mutant isocitrate dehydrogenase (IDH) enzymes characteristic of some cancers. An important theme is emerging whereby metabolism can have a major influence on the epigenetic state (57). The McKenney and Levine Review discusses the clinical aspects of IDH-mutant acute myeloid leukemia and the relationship between altered metabolism and epigenetic changes that impair differentiation in malignant cells (58). Magnetic resonance spectroscopy is a way to noninvasively image metabolites and could be a solution to the challenge of tracking metabolism in patient tumors. Andronesi et al. describe the use of this approach to monitor 2-HG in patients with IDH-mutant cancers (59).

\section{Past successes inform future prospects}

Many drugs originally developed as cytotoxic therapies were recognized later to have specific targets in metabolism (25). The optimal use of these drugs in the clinic was determined empirically, and exactly how these drugs kill cancer cells is still debated. Nevertheless, drugs such as antifolates and nucleoside analogs are mainstays of therapy for many cancers, yet have limited efficacy in other malignancies. In most cases this selectivity for specific cancer types, as well as the variability in response of potentially sensitive tumors, is incompletely understood. Toxicity limits the use of these drugs in some cases, but the fact that a therapeutic window exists for many patients supports the notion that enzymes involved in cell metabolism can be safely targeted for cancer therapy.

Whether novel approaches to target cancer metabolism will be successful in patients remains an open question, but drugs targeting metabolic enzymes will soon be available in the clinic. We can maximize the impact of these new therapies if we embrace the complex issues discussed in this series. For example, how does increased glucose metabolism promote cell proliferation? What role is played by nutrients other than glucose, and under what conditions is use of alternate fuels important? Is there sufficient redundancy in normal tissue metabolism to provide a therapeutic window when some pathways are interrupted? What combination of genetic information, metabolite biomarker levels, and tissue diagnosis is optimal for patient selection? How should patients be monitored for treatment response?

Metabolism unquestionably influences cancer development and progression. The explosion of research to understand cancer cell nutrient utilization will continue to suggest new potential targets. To determine whether this knowledge can be translated into better therapies, we must focus on and further investigate these complexities and challenges in the field moving forward.

\section{Acknowledgments}

I thank members of the Vander Heiden laboratory for thoughtful comments on the manuscript and acknowledge support from the Burroughs Wellcome Fund, the Damon Runyon Cancer Research Foundation, the Smith Family, the Stern Family, the Lustgarten Foundation, and the NIH.

Address correspondence to: Matthew G. Vander Heiden, Koch Institute at MIT, 77 Massachusetts Ave, 76-561, Cambridge, Massachusetts 02139, USA. Phone: 617.715.4471; Fax: 617.253.3189; E-mail:mvh@mit.edu.
1. Metallo CM, Vander Heiden MG. Understanding metabolic regulation and its influence on cell physiology. Mol Cell. 2013;49(3):388-398.

2. Cairns RA, Harris IS, Mak TW. Regulation of cancer cell metabolism. Nat Rev Cancer. 2011;11(2):85-95.

3. Ward PS, Thompson CB. Metabolic reprogramming: a cancer hallmark even warburg did not anticipate. Cancer Cell. 2012;21(3):297-308.

4. $\mathrm{Hu} \mathrm{J}$, et al. Heterogeneity of tumor-induced gene expression changes in the human metabolic network. Nat Biotechnol. 2013;31(6):522-529.

5. Koppenol WH, Bounds PL, Dang CV. Otto Warburg's contributions to current concepts of cancer metabolism. Nat Rev Cancer. 2011;11(5):325-337.

6. Vander Heiden MG, Cantley LC, Thompson CB. Understanding the Warburg effect: the metabolic requirements of cell proliferation. Science. 2009;324(5930):1029-1033.

7. Warburg O. On the origin of cancer cells. Science. 1956;123(3191):309-314.

8. Gatenby RA, Gillies RJ. Why do cancers have high aerobic glycolysis? Nat Rev Cancer. 2004;4(11):891-899.

9. Zu XL, Guppy M. Cancer metabolism: facts, fantasy, and fiction. Biochem Biophys Res Commun. 2004;313(3):459-465.

10. Lunt SY, Vander Heiden MG. Aerobic glycolysis: meeting the metabolic requirements of cell proliferation. Annu Rev Cell Dev Biol. 2011;27:441-464.

11. DeBerardinis RJ, et al. Beyond aerobic glycolysis: transformed cells can engage in glutamine metabolism that exceeds the requirement for protein and nucleotide synthesis. Proc Natl Acad Sci U S A. 2007;104(49):19345-19350.
12. Metallo CM, et al. Reductive glutamine metabolism by IDH1 mediates lipogenesis under hypoxia. Nature. 2012;481(7381):380-384.

13. Mullen AR, et al. Reductive carboxylation supports growth in tumour cells with defective mitochondria. Nature. 2012;481(7381):385-388.

14 . Wise DR, et al. Hypoxia promotes isocitrate dehydrogenase-dependent carboxylation of alpha-ketoglutarate to citrate to support cell growth and viability. Proc Natl Acad Sci U S A. 2011;108(49):19611-19616.

15 . Scott DA, et al. Comparative metabolic flux profiling of melanoma cell lines: beyond the Warburg effect. J Biol Chem. 2011;286(49):42626-42634.

16. Jain M, et al. Metabolite profiling identifies a key role for glycine in rapid cancer cell proliferation. Science. 2012;336(6084):1040-1044.

17. Kamphorst JJ, et al. Hypoxic and Ras-transformed cells support growth by scavenging unsaturated fatty acids from lysophospholipids. Proc Natl Acad Sci U S A. 2013;110(22):8882-8887

18. Commisso C, et al. Macropinocytosis of protein is an amino acid supply route in Ras-transformed cells. Nature. 2013;497(7451):633-637.

19. Maddocks OD, et al. Serine starvation induces stress and p53-dependent metabolic remodelling in cancer cells. Nature. 2013;493(7433):542-546.

20. Zhang WC, et al. Glycine decarboxylase activity drives non-small cell lung cancer tumor-initiating cells and tumorigenesis. Cell. 2012;148(1-2):259-272.

21 . Liu W, et al. Reprogramming of proline and glutamine metabolism contributes to the proliferative and metabolic responses regulated by oncogenic transcription factor c-MYC. Proc Natl Acad SciUS A.
2012;109(23):8983-8988.

22. Hawkins RA, Phelps ME. PET in clinical oncology. Cancer Metastasis Rev. 1988;7(2):119-142.

23. Ben-Haim S, Ell P. 18F-FDG PET and PET/CT in the evaluation of cancer treatment response. J Nucl Med. 2009;50(1):88-99.

24. Geus-Oei LF, Oyen WJ. Predictive and prognostic value of FDG-PET. Cancer Imaging. 2008;8:70-80.

25. Vander Heiden MG. Targeting cancer metabolism: a therapeutic window opens. Nat Rev Drug Discov. 2011;10(9):671-684.

26. Groves AM, Win T, Haim SB, Ell PJ. Non- $\left[{ }^{18} \mathrm{~F}\right]$ FDG PET in clinical oncology. Lancet Oncol. 2007;8(9):822-830.

27. Dimitrakopoulou-Strauss A, Strauss LG. PET imaging of prostate cancer with ${ }^{11} \mathrm{C}$-acetate. J Nucl Med. 2003;44(4):556-558.

28. Baek S, et al. Exploratory clinical trial of (4S)-4-(3$\left[{ }^{18} \mathrm{~F}\right]$ fluoropropyl)-L-glutamate for imaging $\mathrm{xC}$ transporter using positron emission tomography in patients with non-small cell lung or breast cancer. Clin Cancer Res. 2012;18(19):5427-5437.

29. Baek S, et al. (4S)-4-(3-18 F-fluoropropyl)-L-glutamate for imaging of $\mathrm{xC}$ transporter activity in hepatocellular carcinoma using PET: preclinical and exploratory clinical studies. J Nucl Med. 2013;54(1):117-123.

30. Koglin N, et al. Specific PET imaging of $x C$ - transporter activity using a (1)(8)F-labeled glutamate derivative reveals a dominant pathway in tumor metabolism. Clin Cancer Res. 2011;17(18):6000-6011.

31. Lieberman BP, et al. PET imaging of glutaminolysis in tumors by $18 \mathrm{~F}-(2 \mathrm{~S}, 4 \mathrm{R}) 4$-fluoroglutamine. J Nucl 
Med. 2011;52(12):1947-1955.

32. Yuneva MO, et al. The metabolic profile of tumors depends on both the responsible genetic lesion and tissue type. Cell Metab. 2012;15(2):157-170.

33. Son J, et al. Glutamine supports pancreatic cancer growth through a KRAS-regulated metabolic pathway. Nature. 2013;496(7443):101-105.

34. Ying H, et al. Oncogenic Kras maintains pancreatic tumors through regulation of anabolic glucose metabolism. Cell. 2012;149(3):656-670.

35. Guo JY, et al. Autophagy suppresses progression of K-ras-induced lung tumors to oncocytomas and maintains lipid homeostasis. Genes Dev. 2013;27(13):1447-1461.

36. Schulze A, Harris AL. How cancer metabolism is tuned for proliferation and vulnerable to disruption. Nature. 2012;491(7424):364-373.

37. Yun J, et al. Glucose deprivation contributes to the development of KRAS pathway mutations in tumor cells. Science. 2009;325(5947):1555-1559.

38. Wise DR, et al. Myc regulates a transcriptional program that stimulates mitochondrial glutaminolysis and leads to glutamine addiction. Proc Natl Acad Sci U S A. 2008;105(48):18782-18787.

39. Gao P, et al. c-Myc suppression of miR-23a/b enhances mitochondrial glutaminase expression and glutamine metabolism. Nature. 2009; 458(7239):762-765.

40. Jones RG, Thompson CB. Tumor suppressors and cell metabolism: a recipe for cancer growth. Genes Dev. 2009;23(5):537-548.

41. Sonveaux P, et al. Targeting lactate-fueled respira- tion selectively kills hypoxic tumor cells in mice. J Clin Invest. 2008;118(12):3930-3942.

42. Kung HN, Marks JR, Chi JT. Glutamine synthetase is a genetic determinant of cell type-specific glutamine independence in breast epithelia. PLoS Genet. 2011;7(8):e1002229.

43. Pellerin L, Magistretti PJ. Glutamate uptake into astrocytes stimulates aerobic glycolysis: a mechanism coupling neuronal activity to glucose utilization. Proc Natl Acad Sci U S A. 1994;91(22):10625-10629.

44. Sotgia F, Martinez-Outschoorn UE, Howell A, Pestell RG, Pavlides S, Lisanti MP. Caveolin-1 and cancer metabolism in the tumor microenvironment: markers, models, and mechanisms. Annu Rev Pathol. 2012;7:423-467.

45. Pollak M. Insulin and insulin-like growth factor signalling in neoplasia. Nat Rev Cancer. 2008;8(12):915-928.

46. Dewys WD, et al. Prognostic effect of weight loss prior to chemotherapy in cancer patients. Eastern Cooperative Oncology Group. Am J Med. 1980;69(4):491-497.

47. Martin L, et al. Cancer cachexia in the age of obesity: skeletal muscle depletion is a powerful prognostic factor, independent of body mass index. J Clin Oncol. 2013;31(12):1539-1547.

48. Evans JM, Donnelly LA, Emslie-Smith AM, Alessi DR, Morris AD. Metformin and reduced risk of cancer in diabetic patients. BMJ. 2005; 330(7503):1304-1305.

49. Bowker SL, Majumdar SR, Veugelers P, Johnson JA Increased cancer-related mortality for patients with type 2 diabetes who use sulfonylureas or insulin. Diabetes Care. 2006;29(2):254-258.

50. Pollak M. Potential applications for biguanides in oncology. J Clin Invest. 2013;123(9):3693-3700.

51. Semenza GL. HIF-1 mediates metabolic responses to intratumoral hypoxia and oncogenic mutations. J Clin Invest. 2013;123(9):3664-3671.

52. Hensley CT, Wasti AT, DeBerardinis RJ. Glutamine and cancer: cell biology, physiology, and clinical opportunities. J Clin Invest. 2013; 123(9):3678-3684.

53. Doherty JR, Cleveland JL. Targeting lactate metabolism for cancer therapeutics. J Clin Invest. 2013;123(9):3685-3692.

54. Yang M, Soga T, Pollard PJ. Oncometabolites: linking altered metabolism with cancer. J Clin Invest. 2013;123(9):3652-3658.

55 . Ternette $\mathrm{N}$, et al. Inhibition of mitochondrial aconitase by succination in fumarate hydratase deficiency. Cell Rep. 2013;3(3):689-700.

56. Sullivan LB, et al. The proto-oncometabolite fumarate binds glutathione to amplify ROS-dependent signaling. Mol Cell. 2013;51(2):236-248.

57. Kaelin WG, McKnight SL. Influence of metabolism on epigenetics and disease. Cell. 2013;153(1):56-69.

58. McKenney AS, Levine RL. Isocitrate dehydrogenase mutations in leukemia. J Clin Invest. 2013;123(9):3672-3677.

59. Andronesi OC, et al. Detection of oncogenic IDH1 mutations using magnetic resonance spectroscopy of 2-hydroxyglutarate. J Clin Invest. 2013;123(9):3659-3663. 